\title{
Visual Information Present in Infragranular Layers of Mouse Auditory Cortex
}

\author{
Ryan J. Morrill ${ }^{1,2,3}$ and Andrea R. Hasenstaub ${ }^{1,2,3}$ \\ ${ }^{1}$ Coleman Memorial Laboratory, ${ }^{2}$ Neuroscience Graduate Program, and ${ }^{3}$ Department of Otolaryngology-Head and Neck Surgery, University of \\ California-San Francisco, San Francisco, California 94158
}

The cerebral cortex is a major hub for the convergence and integration of signals from across the sensory modalities; sensory cortices, including primary regions, are no exception. Here we show that visual stimuli influence neural firing in the auditory cortex of awake male and female mice, using multisite probes to sample single units across multiple cortical layers. We demonstrate that visual stimuli influence firing in both primary and secondary auditory cortex. We then determine the laminar location of recording sites through electrode track tracing with fluorescent dye and optogenetic identification using layer-specific markers. Spiking responses to visual stimulation occur deep in auditory cortex and are particularly prominent in layer 6 . Visual modulation of firing rate occurs more frequently at areas with secondary-like auditory responses than those with primary-like responses. Auditory cortical responses to drifting visual gratings are not orientation-tuned, unlike visual cortex responses. The deepest cortical layers thus appear to be an important locus for cross-modal integration in auditory cortex.

Key words: audiovisual; cortical; cross-modal; laminar; multisensory; sensory

\section{Significance Statement}

The deepest layers of the auditory cortex are often considered its most enigmatic, possessing a wide range of cell morphologies and atypical sensory responses. Here we show that, in mouse auditory cortex, these layers represent a locus of cross-modal convergence, containing many units responsive to visual stimuli. Our results suggest that this visual signal conveys the presence and timing of a stimulus rather than specifics about that stimulus, such as its orientation. These results shed light on both how and what types of cross-modal information is integrated at the earliest stages of sensory cortical processing.

\section{Introduction}

The cerebral cortex enables dynamic, flexible responses to the sensory environment. To achieve this, signals from a variety of sources must come together, often across the sensory modalities. For example, the acoustic signal (auditory domain) and the lip movements of speech (visual domain) are integrated to fundamentally influence perception of speech (McGurk and MacDonald, 1976), a process that occurs, at least in part, in the cortex (Skipper et al., 2007). Such sensory information is often viewed as traveling through a cortical hierarchy, from primary regions

Received 0ct. 25, 2017; revised Jan. 18, 2018; accepted Feb. 4, 2018.

Author contributions: R.J.M. and A.R.H. designed research; R.J.M. performed research; R.J.M. and A.R.H. analyzed data; R.J.M. and A.R.H. wrote the paper.

This work was supported by the National Science Foundation GRFP to R.J.M., National Institutes of Health Grant R01DC014101 to A.R.H., the Klingenstein Foundation to A.R.H., Hearing Research Inc. to A.R.H., and the Coleman Memorial Fund to A.R.H.

The authors declare no competing financial interests.

Correspondence should be addressed to Dr. Andrea R. Hasenstaub, Coleman Memorial Laboratory, University of California-San Francisco, San Francisco, CA 94158. E-mail: andrea.hasenstaub@ucsf.edu.

DOI:10.1523/JNEUROSCI.3102-17.2018

Copyright $\odot 2018$ the authors $\quad 0270-6474 / 18 / 382854-09 \$ 15.00 / 0$ through secondary regions and eventually to association cortex. Traditionally, the earliest stages of this processing were thought to be exclusively unimodal, exhibiting responses to only one sensory modality (Felleman and Van Essen, 1991). Recently, this notion has been challenged by evidence that even the primary regions of visual, auditory, and somatosensory cortex all receive and integrate information from other sensory modalities (Calvert et al., 1997; Foxe et al., 2000; Ghazanfar et al., 2005; Schaefer et al., 2006; Iurilli et al., 2012).

Evidence for multisensory convergence in early sensory cortex comes from two complementary lines of research. First, anatomical tracing has revealed direct connections between cortical or thalamic regions of different sensory systems (e.g., Falchier et al., 2010; Banks et al., 2011; Henschke et al., 2015). Second, physiological studies have shown that neural activity, as measured by firing rate or field potential response, can be altered in unimodal versus multimodal stimulus conditions. For example, neural responses to sound in both core and belt regions of monkey auditory cortex (ACtx) can be modulated by the presence of concurrent visual stimulation (Ghazanfar et al., 2005; Kayser et al., 2008). Less common is the finding that a neuron would respond 
to a different sensory modality in absence of stimulation in the modality preferred by the surrounding cortex. In the ACtx, spiking responses to visual stimuli have been shown to develop after behavioral training; specifically, neurons in the ACtx of primates, trained on an auditory categorization task, exhibit responses to the onset of a cue light (Brosch et al., 2005). In the untrained context, ACtx responses to visual stimulation have been found in rats (Wallace et al., 2004), ferrets (Bizley et al., 2007), and gerbils (Kobayasi et al., 2013) but are generally reported to represent a small fraction of responses. Furthermore, the stimulus preferences and cortical organization of these visually responsive neurons remain poorly understood. Such information is critical for understanding the role that these responses play in auditory processing.

Here we sought to determine whether and how the ACtx of the mouse (Mus musculus) responds to visual stimulation. We performed acute, awake recordings in mouse auditory and visual cortices during auditory and visual stimulation. In a subset of recording sites, neurons in mouse ACtx responded to visual stimulation even without a concurrent auditory stimulus. These neurons reside almost exclusively in layer 5 (L5) and layer 6 (L6) of the cortex, and may signal the presence and timing of a salient visual stimulus to the local circuitry of the ACtx.

\section{Materials and Methods}

Animals. All experiments were approved by the Institutional Animal Care and Use Committee at the University of California, San Francisco. For optogenetic identification of L6, we used the Ntsr1-Cre knock-in line (GENSAT GN220), in which Cre recombinase is expressed specifically in L6 corticothalamic cells (Gong et al., 2007; Olsen et al., 2012). To achieve targeted activation of L6, this line was crossed with the Ai32 strain (JAX stock \#012569), which encodes the light-gated depolarizing cation channel channelrhodopsin-2 (ChR2) conjugated to eYFP, after a floxed stop cassette under the CAG promoter. For all other experiments characterizing the visual response in ACtx, we used mouse strains on a C57BL/6 background that were not expressing optogenetic effector proteins. Mice in all experiments were between 6 and 12 weeks old. All adult mice were housed in groups of $2-5$ under a $12 \mathrm{~h} / 12 \mathrm{~h}$ light/dark cycle. Both female (5 of 19) and male mice were included in this study.

In vivo awake recordings. A surgery to implant a custom steel headplate over the temporal skull using dental cement was conducted 2-7 $\mathrm{d}$ before each recording. The headplate was positioned to allow access to a point putatively centered on primary ACtx, $2.5 \mathrm{~mm}$ posterior to bregma and under the squamosal ridge. On the day of the recording, the animal was anesthetized using isoflurane and a $\sim 2$-mm-diameter opening was made in the skull over ACtx using a dental drill. This opening was promptly covered with silicone elastomer (Kwik-Cast, World Precision Instruments), and the animal was allowed to recover from anesthesia for $1-2 \mathrm{~h}$. The animal was then affixed by its headplate over a free-spinning spherical treadmill and the silicone plug over the craniotomy was removed. A 16-channel linear probe ( $50 \mu \mathrm{m}$ site spacing; Neuronexus) was covered in the lipophilic dye $\mathrm{Di}-\mathrm{I}(2.5 \mathrm{mg} / \mathrm{ml}$ in EtOH) using a needle and syringe and then slowly inserted in the brain using a motorized microdrive (FHC). After reaching the desired depth, the brain was allowed to settle for $\sim 10 \mathrm{~min}$, after which neural recording and stimulus presentation commenced. Typically, 3-5 acute penetrations were performed per animal.

The signal acquisition system consisted of an Intan RHD2000 recording board and an RHD2132 amplifier (Intan Technologies), sampling at $30 \mathrm{kHz}$. Auditory stimuli were presented with a free-field electrostatic speaker (ES1, Tucker-Davis Technologies) driven by a Quad Capture external soundcard (Roland) at a sampling rate of $192 \mathrm{kHz}$. Visual stimuli were presented on a 19 inch LCD monitor with a $60 \mathrm{~Hz}$ refresh rate (VW199, ASUS). Auditory and visual stimuli were both generated in MATLAB using the Psychophysics Toolbox Version 3 (Kleiner et al., 2007).
Sound stimuli consisted of blocks of click trains followed by pure tone sequences. Click trains, generated from broadband $5 \mathrm{~ms}$ white noise pulses, were presented at $20 \mathrm{~Hz}$ for $500 \mathrm{~ms}$ duration, and were used as a search stimulus to determine auditory responsiveness; they were not analyzed further. Pure tone stimuli consisted of $100 \mathrm{~ms}$ tones of varied frequencies $(4-64 \mathrm{kHz}, 0.2$ octave spacing) and sound attenuation levels (30-60 dB in $5 \mathrm{~dB}$ linear steps), with an interstimulus interval of $500 \mathrm{~ms}$ to construct a frequency-response area (FRA). Between 6 and 10 trials were presented at each frequency-attenuation level.

Visual stimuli consisted of either flash stimuli or drifting grating stimuli on a monitor centered in front of and $25 \mathrm{~cm}$ away from the mouse. Monitor luminance was calibrated to $25 \mathrm{~cd} / \mathrm{m}^{2}$ for a gray screen, measured at the approximate location of the mouse's eyes. The flash stimulus was a white square $\left(32^{\circ}\right.$ horizontal $\times 32^{\circ}$ vertical $)$ on a black background, $150 \mathrm{~ms}$ in duration, with a peak brightness of $95 \mathrm{~cd} / \mathrm{m}^{2}$. Typically, 150 flash presentations were used per block, and the interstimulus interval was randomly varied between 650 and $2850 \mathrm{~ms}$. Drifting gratings were presented full screen $\left(79^{\circ}\right.$ horizontal $\times 50^{\circ}$ vertical $)$ for $1 \mathrm{~s}$ using parameters optimal for driving mouse visual cortex (VCtx): $4 \mathrm{~Hz}$ temporal frequency, 0.02 cycles/degree spatial frequency, $100 \%$ contrast (Niell and Stryker, 2008). Gratings were presented in 12 orientations from 0 to 330 degrees in a randomly varied sequence, with 50 presentations per orientation and a randomly varied interstimulus interval between 500 and $1600 \mathrm{~ms}$. To verify that the monitor did not produce sound, we recorded and analyzed sounds during visual stimulus presentation using an ultrasonic microphone and recording device sampling at $250 \mathrm{kHz}$ (UltraSoundGate 416H, Avisoft Bioacoustics).

For L6 optogenetic identification experiments, we activated ChR2 by illuminating the cortex with a blue $470 \mathrm{~nm}$ LED (Mightex) coupled to a $400-\mu \mathrm{m}$-diameter optical fiber, $\mathrm{NA}=0.39$ (Thorlabs). A micromanipulator was used to position the fiber tip just above the cortical surface immediately adjacent to the probe penetration site. Light powers were varied between 0.2 and $2.2 \mathrm{~mW}$; trials with light powers of $1.6-2.2 \mathrm{~mW}$ were used for later analysis. Light duration was $500 \mathrm{~ms}$, with a $50 \mathrm{~ms}$ linear ramp to reach full power and a recovery time randomly varied between 1600 and $2600 \mathrm{~ms}$.

Data analysis. After recordings, the raw voltage trace was bandpassed between $600 \mathrm{~Hz}$ and $6 \mathrm{kHz}$, and events were extracted using a movingwindow $4.5 \mathrm{SD}$ threshold. For single unit (SU) analysis, event waveforms were sorted using custom software in MATLAB (KFMMAutoSorter, written by Mathew Fellows). Multiunit (MU) analysis was performed on all events captured by the $4.5 \mathrm{SD}$ threshold; as such, this analysis includes all units recorded on a channel, as well as events that could not be attributed to a SU neural source due to the absence of a uniquely identifiable waveform shape. Such analysis is typically thought to capture spiking activity from tens of neurons in the vicinity of the recording electrode (Buzsáki, 2004).

For all MU and SU analyses, auditory responsiveness was defined as a significant difference in firing rate between the $100 \mathrm{~ms}$ before stimulus onset and the $100 \mathrm{~ms}$ poststimulus period (paired $t$ test, BenjaminiHochberg corrected for false discovery rate, $q=0.001$ ) (Benjamini and Hochberg, 1995). The Benjamini-Hochberg procedure, used here to correct for multiple comparisons in determining significance of auditory and visual responses, is a method for controlling false positives (Type I errors) that has increased power relative to more common family-wise error rate control procedures, such as the Bonferroni correction; the latter class of procedures attempts to control the probability of including one false positive, typically at the expense of a high false negative (Type II error) rate. On the other hand, false discovery rate methods set an acceptable rate of Type I errors (Benjamini and Hochberg, 1995). Here, we set our false discovery rate for both auditory and visual responses to $q=$ 0.001. Auditory FRAs were generated using firing rate in the $100 \mathrm{~ms}$ after sound onset. Significant tuning to frequency, used in auditory response classification, was defined as a modulation of firing rate by frequency using a one-way ANOVA $(\alpha=0.05)$.

Visual responsiveness was defined as a significant difference in firing rate between the $200 \mathrm{~ms}$ preceding stimulus onset and $200 \mathrm{~ms}$ poststimulus onset (paired $t$ test, Benjamini-Hochberg corrected for false discovery rate; $q=0.001)$. MU sites and units considered in this dataset were 
from depths within cortex, as measured by our electrode track tracing procedure described below, and only included recordings determined to be in ACtx based on MU responses to pure tones ( $n=676 \mathrm{MU}$ sites; $n=$ 223 SUs).

For both auditory and visual responses, latency to onset was defined as the time point at which the poststimulus firing rate exceeded the prestimulus firing rate by 4 SDs. Likewise, response offset was defined as the point after onset at which firing rate dropped back down $<4$ SDs above prestimulus firing rate.

To identify the Ntsr 1-Cre-positive L6 band in our Ai32/Ntsr1-Cre optogenetic activation experiments, we analyzed recordings for a group of three or more adjacent light-activated channels. A channel was defined as light-activated if it showed a sustained increase in MU firing rate throughout the light-on period. To remove transient onset effects that were often observed throughout the cortical column, our analysis focused on the last $200 \mathrm{~ms}$ of the light-on period. We converted firing rate during this period to a $z$ score using the baseline $(200 \mathrm{~ms}$ before stimulus onset) mean and SD. Degree of firing rate modulation differed greatly between recordings, presumably because of factors such as light penetrance in cortical tissue. As such, we defined significant activation as any period that surpassed half of the peak $z$ score firing rate observed across all light levels in each recording. A channel was considered light responsive if at least half of all $20 \mathrm{~ms}$ time bins showed such activation, and an Ntsr1-Cre band was defined as three adjacent channels (i.e., activation spanning $150 \mu \mathrm{m}$ ) Using this method, the Ntsr1-Cre-positive band of L6 was readily identifiable in 5 of 8 experiments with visually responsive SUs or MUs.

To determine the degree of tuning to orientation of drifting grating stimuli, we calculated an orientation selectivity index (OSI) as follows:

$$
\text { OSI }=\frac{R_{\text {pref }}-R_{\text {orth }}}{R_{\text {pref }}+R_{\text {orth }}}
$$

In the above equation, $R_{\text {pref }}$ is the mean response to the stimulus of the preferred orientation (that which elicited the response with the highest firing rate), and $R_{\text {orth }}$ is the mean response to the two orientations orthogonal to the preferred orientation. All responses were baselinecorrected by subtracting the mean prestimulus firing rate averaged over all trials. For units in which $R_{\text {orth }}$ was suppressed relative to baseline, OSI will be $>1$. Analyses were performed on the $500 \mathrm{~ms}$ after stimulus onset.

Histological verification of electrode track depth. To visualize recording site locations, we used the fluorescent lipophilic dye Di-I, which has been shown to reliably mark the full extent of electrode tracks in extracellular recordings (DiCarlo et al., 1996) and has been used to visualize multisite silicon probe tracks in the mouse brain (Lee et al., 2015). After completion of physiological recordings, the animal was killed, and the brain was removed and placed into a solution of $4 \%$ PFA in PBS $(0.1 \mathrm{M}, \mathrm{pH} 7.4)$ for $12 \mathrm{~h}$, followed by $30 \%$ sucrose in PBS solution for several days. The brain was then frozen, and sections were cut on a sliding microtome (SM2000R, Leica Biosystems). Slices were then mounted and imaged on a fluorescence microscope with a red filter cube (Eclipse 90i, Nikon). Fluorescent marks on slices were mapped to each recording with the aid of a penetration site map drawn based on the exposed cortical surface during the experiment. Approximately half of the electrode tracks were fully imaged and mapped onto the Paxinos and Franklin (2004) for 3D reconstruction of recording site location. For the remainder, histology was used only to identify the depth of recorded electrode sites and verify that they were located in the cortex.

Classification of auditory sites. ACtx contains subfields with characteristic neural responses to sound stimuli (Stiebler et al., 1997; Joachimsthaler et al., 2014). We classified recording sites as either primary-like in their responses (putatively primary ACtx, A1, or anterior auditory field) or secondary-like (for example, secondary ACtx, A2, or dorsal-posterior field). Primary and secondary regions are most differentiable by latency to response onset, with primary regions exhibiting shorter onset latencies than secondary ones (Carrasco and Lomber, 2011; Joachimsthaler et al., 2014). As such, the classification procedure we used was as follows. A channel-wise automatic classification was made for MU pure tone responses based on the following criteria: primary-like responses were those with significant firing rate tone responses and onset latencies of $<14$ ms; secondary-like responses were those with significant tone responses and onset latencies of $>14 \mathrm{~ms}$ (Joachimsthaler et al., 2014). Channels not significantly responsive to sound were coded as nonauditory. Next, channels whose classification differed from that of their neighbors were examined in the context of all auditory responses on the probe and coded by eye. This dealt with two problematic cases resulting from automatic channel-wise classification. First, this allowed us to correct for "one-off" inconsistencies in classification within a probe. Second, this increased labeling accuracy in border cases where the probe track did not enter orthogonal to the brain surface, and both primary- and secondary-like responses were recorded from the same auditory experiment. All auditory response classification was performed blind to results of the visual response experiments conducted at the same site.

\section{Results}

\section{Visual responses in mouse ACtx}

To measure visual responses in mouse auditory cortical neurons, we performed acute extracellular recordings in awake mice using a linear probe to simultaneously measure responses in different layers of cortex (Fig. 1A). In separate blocks, mice were either presented with $100 \mathrm{~ms}$ pure tones of varied frequencies and attenuations or $150 \mathrm{~ms}$ flashes of a white square on a black background (Fig. 1B). We then analyzed MU and SU activity evoked by these auditory and visual stimuli (Fig. $1 C$ ). Recording site depth and location were determined from post hoc histological visualization of the lipophilic dye Di-I, which was applied to the probe shank before each penetration (Fig. $1 D, E$ ). Probe placement was targeted to the ACtx based on surface vasculature (Stiebler et al., 1997) and confirmed by robust responsiveness to sound stimulation at the recording sites (see Materials and Methods; Fig. $1 F$, left and middle). After identifying ACtx, we presented flashes and determined whether firing rate was modulated by these purely visual stimuli (Fig. $1 F$, right). In 28 of 48 ACtx laminar recordings from 16 mice, at least one MU showed a statistically significant increase in firing rate in response to the visual stimulus (paired $t$ test, Benjamini-Hochberg corrected for false discovery rate, $q=0.001$; see Materials and Methods). Visually evoked spiking responses were found in both MUs (Fig. $1 G$ ) and SUs (Fig. 1H). Habituation of responses to repeated stimulus presentations is a well-established feature of auditory cortical processing (Cook et al., 1968). We tested whether auditory cortical SU visual responses found here also habituated over the course of stimulus presentation blocks by checking for a systematic increase or decrease in response magnitude using Spearman's correlation analysis. We found 3 of 15 units from 9 mice whose stimulus-evoked firing rates changed (with no corresponding change in baseline firing; $p<0.05$ ). Of these, two decreased in firing rate and one increased. Additionally, we found no trend in stimulus-evoked firing rate on the population level (Jonckheere-Terpstra trend test statistic $=0.84, p=0.20$ ). Together, these tests show that there is little evidence for systematic increase or decrease of response magnitude over time with our recording protocol.

Recordings were performed in a blocked manner, with auditory stimuli presented together followed by visual stimuli. Not all units identified as visually responsive were also identifiable in the auditory blocks, due either to sparse firing in response to auditory stimulation or electrode drift over time. There were 9 wellisolated visually responsive SUs also identified in auditory recordings ( $n=7$ recordings from 7 mice); of these, 7 units ( 7 of 9), exhibited significant auditory responses (example: Fig. 1I), all of which were also tuned to sound frequency. This result 


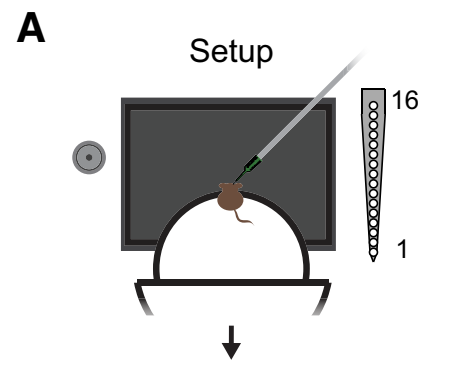

B

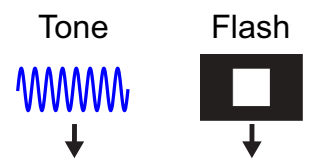

C

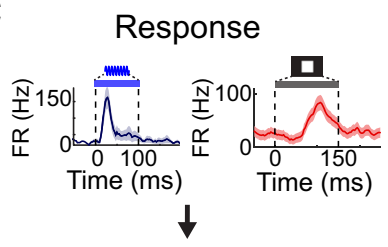

D

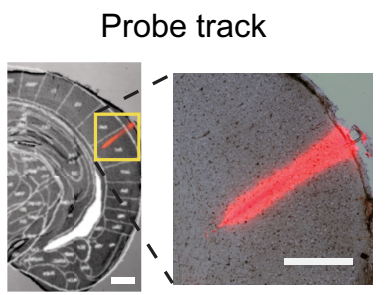

E

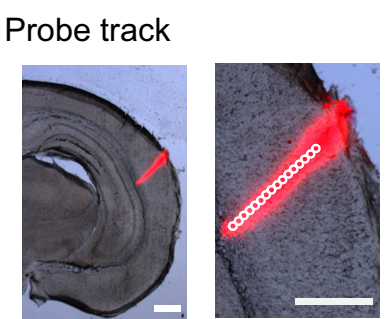

F

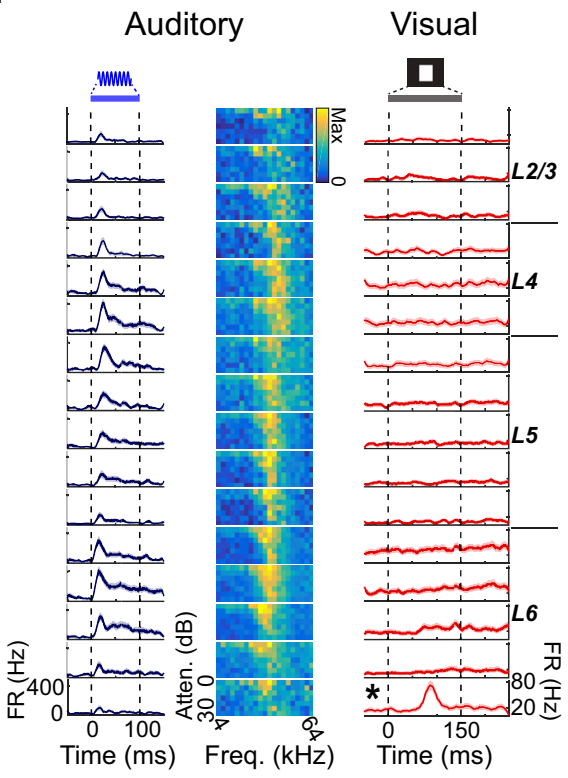

G

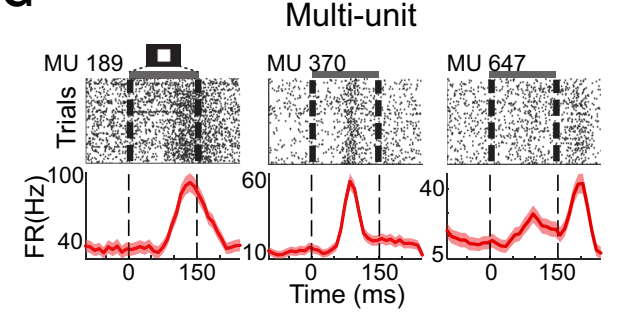

H

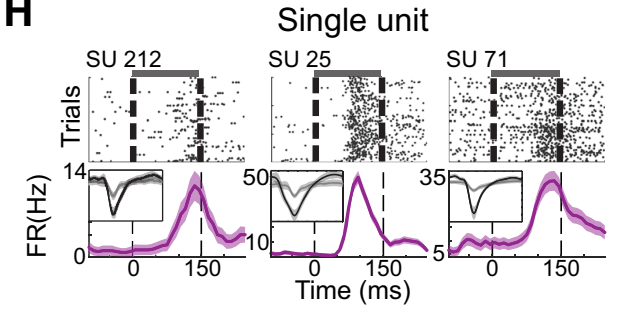

I

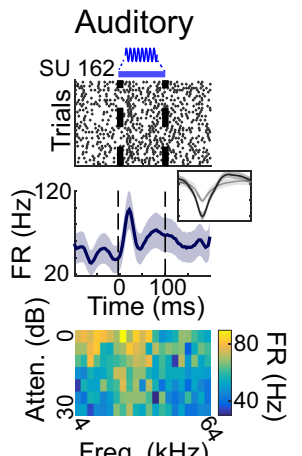

Visual

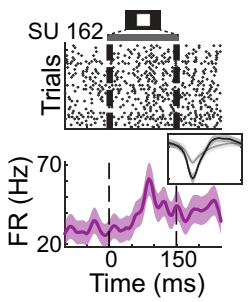

Figure 1. A, Awake, acute recordings in right mouse ACtx using a 16-channel multisite probe (right), experimental sequence: $\boldsymbol{B}-\boldsymbol{D}$. $\boldsymbol{B}$, Tone stimuli used for identification of characteristic auditory cortical responses (left) and flash stimuli to test for visual responsiveness (right). C, Auditory and visual MU responses (mean \pm standard error) on the same recording channel. $\boldsymbol{D}$, Probe location visualized using fluorescent dye Di-I, which was applied to the probe before recording. Images at $1 \times$ magnification (left) and $4 \times$ (right) used to determine location and laminar depth. Scale bar, $500 \mu \mathrm{m}$. E, Probe track marked by Di-I, marked with electrode site locations. White circles represent locations of channels shown in $\boldsymbol{F}$. F, MU responses on all 16 channels from one recording. Left, Responses to tones of BF. Middle, Frequency response area. Right, Response to visual stimulus. *Significant response at $q=0.001$ after Benjamini-Hochberg false discovery rate correction (see Materials and Methods). $\boldsymbol{G}$, Additional examples of statistically significant MU responses to visual stimuli from different mice. $\boldsymbol{H}$, Examples of statistically significant $\mathrm{SU}$ responses to visual stimuli. Inset, Waveforms. Black represents SU. Gray represents MU from corresponding channel. I, Example SU showing both auditory and visual responses.

shows that some neurons in mouse ACtx multiplex auditory and visual stimuli.

\section{Deep layer bias of visual responses in ACtx}

The laminar location of a neural response can be strongly suggestive of its computational role. We mapped the geometry of the probe electrode sites onto brain slice images marked by Di-I (Figs. $1 E$, right, $2 A$ ) to determine the cortical depths of visuallyresponsive electrode sites (example: Fig. $2 B$ ). We then measured the distance from white matter of each recording site and normalized this by the white matter-pia distance of the corresponding brain slice to correct for any tissue distortion. This yielded a fractional cortical depth measurement for each recorded channel, which was then assigned to a cortical layer (from 48 recordings in 16 mice: L6, $n=160$ sites; L5, $n=254$; L4, $n=129$; L2/3, $n=120$; L1, $n=13$; Fig. $2 D$ ). Analysis of these data reveals that the majority of MU and SU visual responses occur in L6, with the bulk of the remainder occurring in L5 (Fig. 2C,D). To provide physiological confirmation of our depth measurements, we used the Ntsr1-Cre mouse strain in which Cre recombinase is expressed specifically in L6 corticothalamic cells (Fig. 2E) (Gong et al., 2007; Olsen et al., 2012). This mouse line was crossed with mice of the Ai32 strain, which expresses ChR2 conjugated to
eYFP in a Cre-dependent manner, so that $\mathrm{ChR} 2$ was restricted to L6 (Fig. 2E). Illumination of the cortical surface with blue light resulted in strong MU activation of a distinct band of channels deep on the probe (Fig. $2 F, G$ ), thereby providing an optogenetically induced physiological marker of L6. A band of three or more adjacent channels with sustained optogenetic activation was identified in five of eight recordings ( $n=4$ of 6 mice). We determined the depth of visual responses relative to this band of activation and found that all of them occurred $\leq 200 \mu \mathrm{m}$ from its lower border (Fig. 2H). This "photo-tagging" approach to identify the band of L6 corticothalamic cells further confirmed the deep layer bias of visual responses.

Visual responses in primary and secondary regions of ACtx The neural signatures of multisensory integration are more commonly observed in secondary or "higher-order" areas of sensory cortex compared with primary regions (Ghazanfar et al., 2005; Bizley et al., 2007). To test whether this finding holds for visual responses in mouse ACtx, we classified our recording sites into primary or secondary regions using temporal dynamics and frequency tuning of evoked MU responses to pure tones of varied frequencies and attenuations (see Materials and Methods). Recordings classified as primary-like typically showed robust 

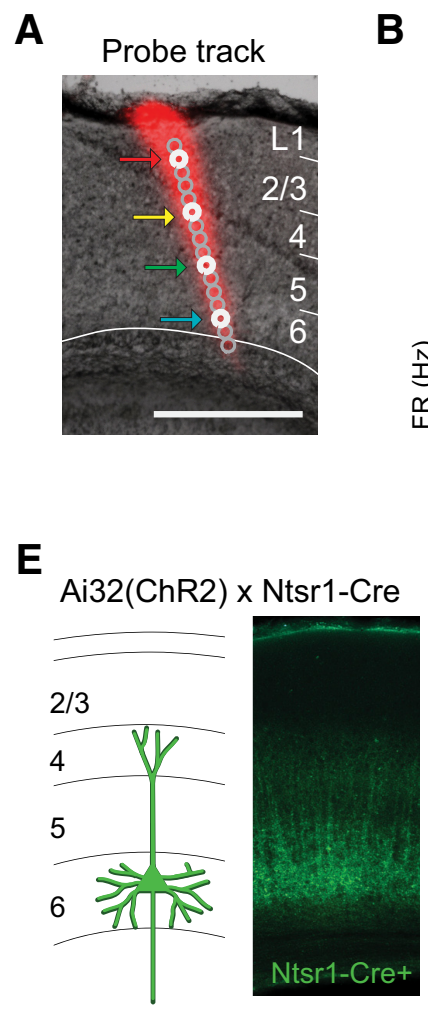

B

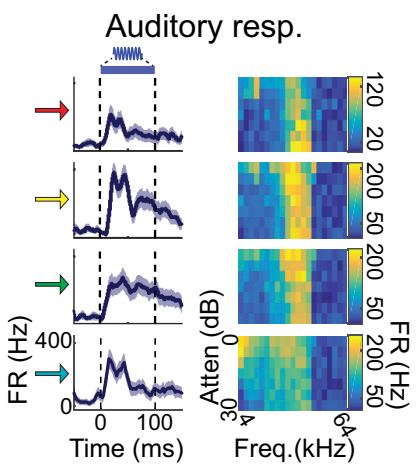

$\mathbf{F}$

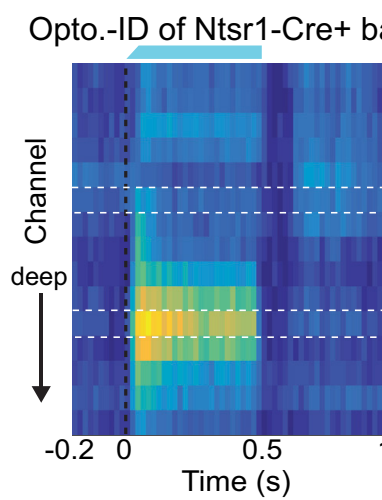

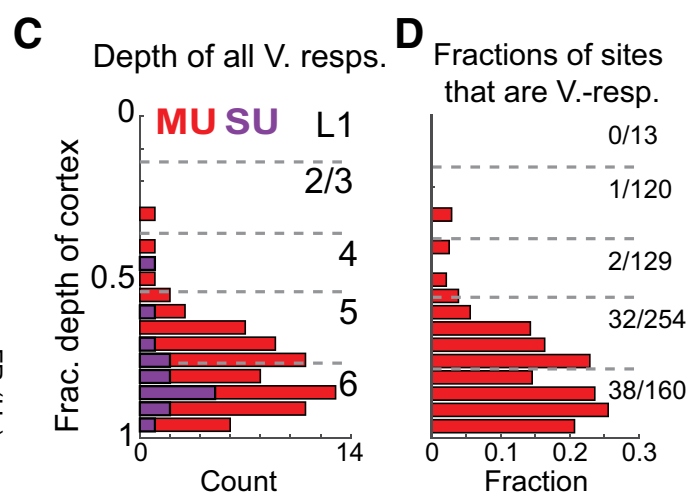

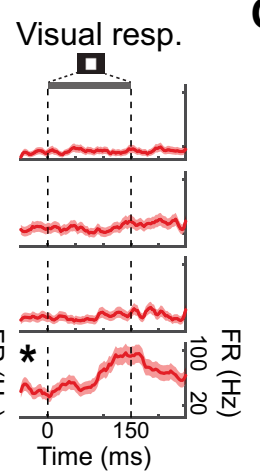

G

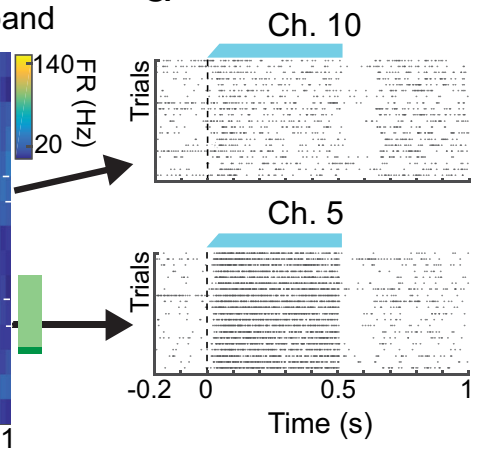

H

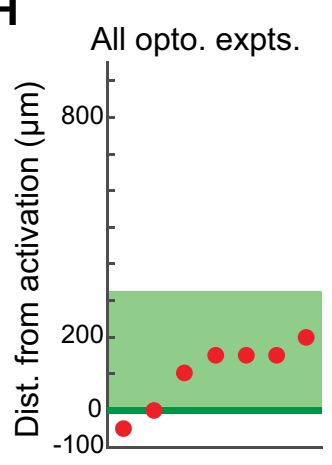

Figure 2. A, Probe track in ACtx visualized with Di-I, electrode sites marked by circles. $\boldsymbol{B}$, Colored arrows indicate channels. Scale bar, $500 \mu \mathrm{m}$. $\boldsymbol{B}$, MU auditory responses (left), frequency response area (middle), and visual responses (right) at sites from cortical depths indicated by colored arrows in $\boldsymbol{A}$. *Visually responsive MU is located in L6. C, All visual responses shown as a function of depth. Red represents MUs. Purple represents SUs. $n=73$ MUs and 15 SUs from 30 recordings in 15 mice. $D$, Fractions of visually responsive sites by depth. Numbers at left indicate total number of MU visual responses over total number of sites recorded in each layer. $\boldsymbol{E}$, Ai32/Ntsr1-Cre mice express eYFP-tagged ChR2 in L6; histology showing eYFP (green) on right. $\boldsymbol{F}$, Representative example of optogenetic identification of $\mathrm{L} 6$ through activation of Ntsr1-Cre-positive cells. MU activity shows a band activated strongly during light-on period (cyan, indicated above). Significantly modulated channels indicated by green band at right. $\mathbf{G}$, Top, Superficial channel shows minimal light-related MU activity. Bottom, Deep channel MU activity shows strong effect of light activation. $\boldsymbol{H}$, Summary plot of all visually responsive MUs (red dots) from all recordings with an identifiable light-activated L 6 band ( $n=7$ MUs from 5 recordings in 4 mice) plotted by depth relative to the lower border of this band (green).

frequency-attenuation tuning (example: Fig. 3A), whereas many sites classified as secondary did not (example: Fig. 3B). When reconstructed, most visually responsive primary-like sites were found within primary ACtx on the Paxinos and Franklin (2004) mouse brain atlas (Fig. 3C). Mean normalized FRAs from primary and secondary sites centered on best frequency $(\mathrm{BF})$ show that, on average, MUs from both classification exhibit tuning, but $\mathrm{BF}$ - and off-BF responses were closer in magnitude in secondary than in primary sites (Fig. 3E). MU onset and peak response latencies to BF sound stimuli in primary areas were lower than those in secondary areas (primary onset: $10 \pm 4 \mathrm{~ms}$, mean $\pm S D$; secondary onset: $17 \pm 11 \mathrm{~ms}$; Wilcoxon rank-sum $Z=11.7, p=$ 1.71e-31; primary peak: $18 \pm 8 \mathrm{~ms}$; secondary peak: $30 \pm 16 \mathrm{~ms}$; rank $\operatorname{sum} Z=9.76, p=1.67 \mathrm{e}-22 ; n=396,167 \mathrm{MUs}$ from $n=32$, 23 , recordings in $n=14,13$ mice for primary and secondary, respectively; Fig. 3D). While distributions of recorded auditory BFs were biased to the middle of the frequency band tested $(\sim 8-30 \mathrm{kHz})$, visual responses were more prominent at those sites with BFs near $64 \mathrm{kHz}$ (Fig. 3F). The fraction of visual responses was also slightly higher at secondary than primary recording sites in both L5 and L6, the only two layers that exhibited any substantial visual responsiveness (Fig. $3 G$ ). Thus, our results indicate that visual MU responses are slightly biased toward secondary sites and toward sites with high-frequency tuning in primary areas, but are nevertheless present at sites with a variety of auditory BFs.

\section{Visual response latencies compared in auditory and visual cortices}

Latencies to response onset for tones at $\mathrm{BF}$ in the ACtx vary from 8 to $30 \mathrm{~ms}$, depending on auditory field (Fig. $3 D$ ). What are the temporal dynamics of the visual response in ACtx? Visually responsive SUs in primary auditory regions exhibit onset latencies of $75 \pm 10 \mathrm{~ms}$ (mean $\pm \mathrm{SD} ; n=7$ SUs from 6 recordings in 6 mice), whereas those in secondary regions have onsets of $92 \pm 25$ $\mathrm{ms}(n=8$ SUs from 5 recordings in 5 mice; Fig. $4 A)$; this difference did not reach statistical significance (Wilcoxon rank-sum $=$ $47.5, p=0.34)$. MU visual response onset latencies were $85 \pm 37$ $\mathrm{ms}$ in primary sites ( $n=45$ MUs from 19 recordings in 12 mice) and $95 \pm 25 \mathrm{~ms}(n=28$ MUs from 11 recordings in 8 mice $)$ in secondary (Fig. 4B). MU visual response onsets in primary ACtx occurred significantly earlier than those in secondary ACtx (Wilcoxon rank-sum $Z=2.3, p=0.021$ ).

Anatomical tracing work has shown that mouse ACtx receives direct inputs from several visual cortical regions, and that these inputs show a preference for L1 and L6 (Banks et al., 2011). If these projections are carrying the visual information to the ACtx, visual stimulation should elicit earlier responses in VCtx than in ACtx. To test this, we recorded from awake mouse VCtx using flash stimuli with the same parameters as used to elicit responses in ACtx (Fig. 4C,D). Analysis of MU data shows that responses to visual stimuli in VCtx are significantly earlier in onset than in ACtx (VCtx: $40 \pm 11 \mathrm{~ms}, n=78$ MUs from 8 recordings in 3 


\section{A}
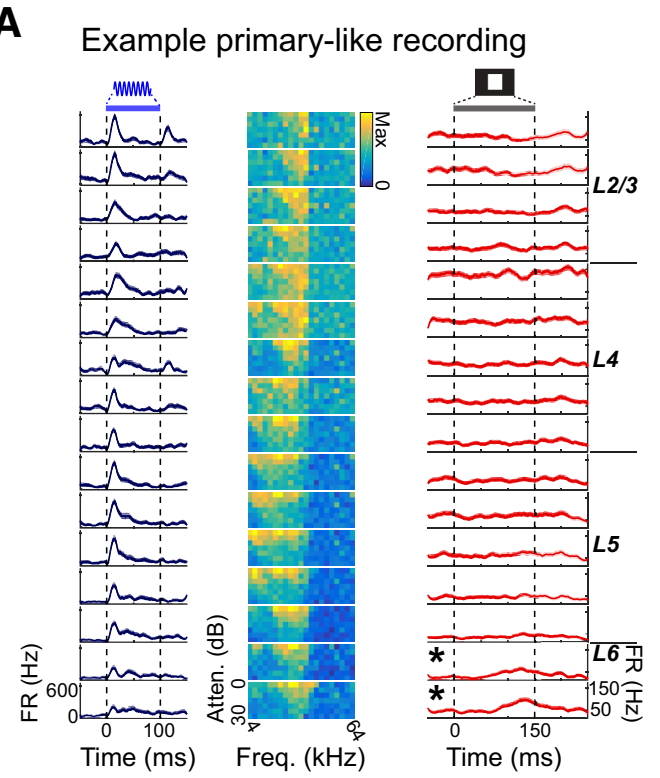

$F$
B

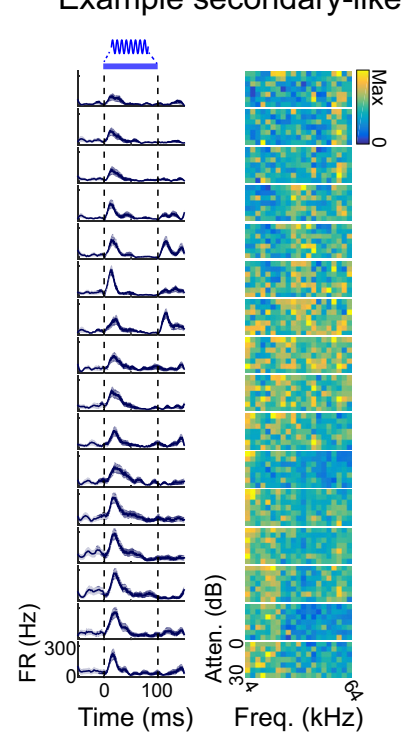

D

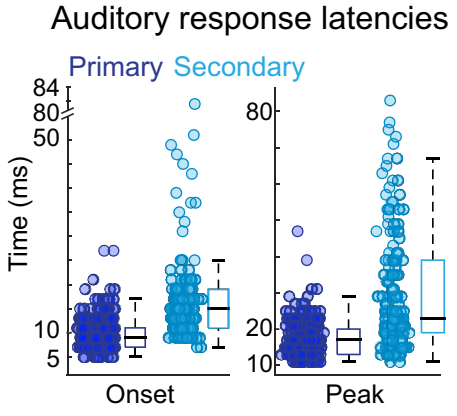

E

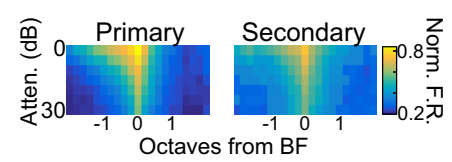

Auditory best frequencies

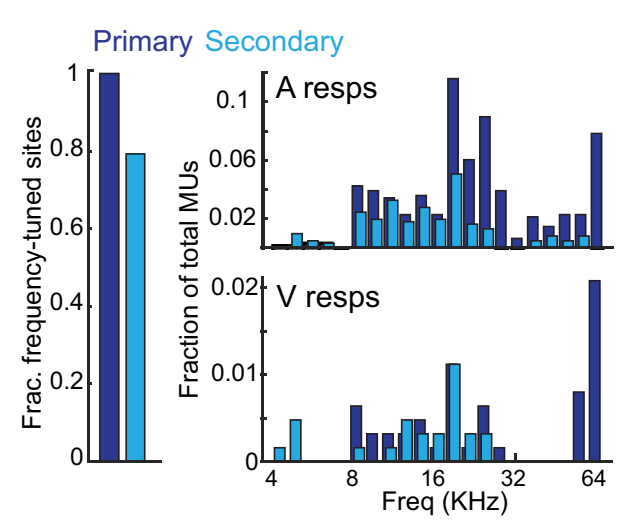

C

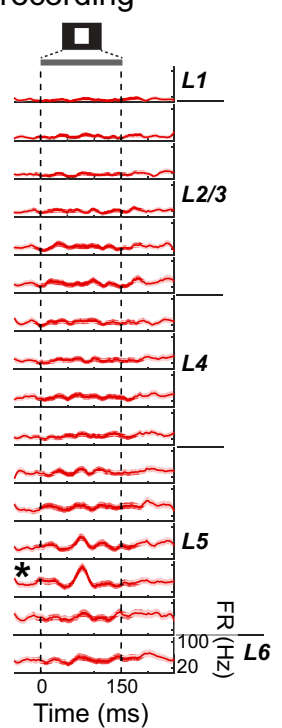

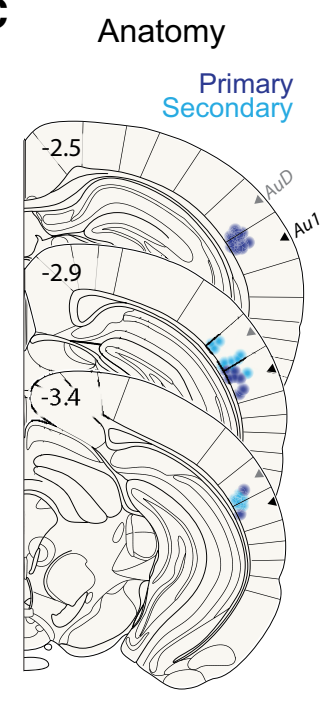

G

Aud. and vis. resps. by layer

Primary Secondary Visual

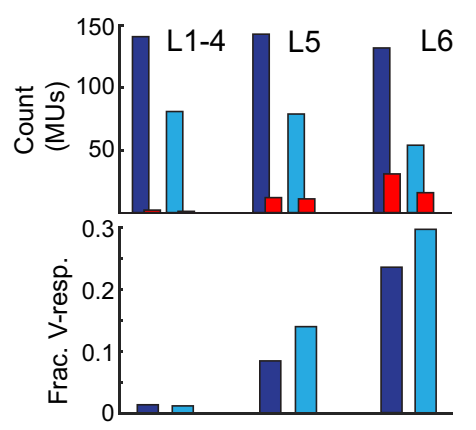

Figure 3. A, Left, Example recording classified as primary-like, based on short latency of responses to tones at BF, all 16 channels shown. Middle, Primary-like sites show a high degree of tuning to frequency and attenuation. Right, Response to visual stimulation at same site. * Responses on deepest two channels were significant at $q=0.001$ (see Materials and Methods). $\boldsymbol{B}$, Recording classified at secondary-like, based on longer latency of responses to tones of $B F$, as in $A$. Significant MU visual response $(q=0.001)$ recorded on channel third from bottom. $C$, Locations of visually responsive channels from anatomically reconstructed sites $(n=13$ mice), color-coded blue ( $n=22$ sites) or cyan ( $n=24$ sites) for primary or secondary classification, respectively (section drawings from Paxinos and Franklin, 2004). Depth and anteroposterior distance determined from electrode track histology (see Fig. 1D). Distance behind the mouse skull landmark bregma and putative positions of primary ACtx (Au1, black) and dorsal ACtx (AuD, gray) are marked on each section. D, MU auditory response latencies by auditory classification; in box plots, black line indicates median, box edges indicate 25th and 75th percentile and whiskers extend to most extreme data points excluding outliers. Latencies at primary-like regions are shorter both in onset (left; Wilcoxon rank-sum $Z=11.75, p=6.50 \mathrm{e}-32$ ) and peak (right; Wilcoxon rank-sum $Z=10.34, p=4.20 \mathrm{e}-25$ ). $E$, Average MU FRA showing tuning from all sites classified as primary (left) or secondary (right), centered on BF. Before averaging, all FRAs were normalized to peak response. $\boldsymbol{F}$, Auditory BF of all auditory-responsive MUs (top) and visually responsive MUs (bottom). $\boldsymbol{G}$, Total counts of all recorded auditoryand visual-responsive MUs by layer (top). Fraction of visually responsive MUs (bottom), showing biases toward deeper layers and sites with secondary-like responses.

mice; ACtx: $90 \pm 32 \mathrm{~ms}, n=73$ MUs from 30 recordings in 15 mice; Wilcoxon rank-sum $Z=9.06, p=1.26 \mathrm{e}-19)$ and peak (VCtx: $70 \pm 33$ ms; ACtx: $115 \pm 39 \mathrm{~ms}$; Wilcoxon rank-sum $Z=$ 7.60, $p=2.95 \mathrm{e}-14)$. Latencies to response offset in VCtx and ACtx were not significantly different (VCtx: $160 \pm 73 \mathrm{~ms}$; ACtx: $131 \pm 40 \mathrm{~ms}$; Wilcoxon rank-sum $Z=1.4 p=0.16$; Fig. $4 E, F$ ), although VCtx sites showed a wider distribution of offset latencies (Fig. 4F). These dynamics show that VCtx begins processing the visual flash stimulus before it arrives in the ACtx.

\section{Visual orientation tuning in the ACtx}

A hallmark of visual cortical processing is tuning of neurons to edges of particular orientations. We sought to test whether visual responses in ACtx also carry specific information about the visual scene, such as edge orientation. While recording in ACtx, we presented full screen $1 \mathrm{~s}$ drifting gratings of 12 orientations and found strong responses in a subset of our flash-responsive SUs (example, Fig. 5A). Comparison of response peristimulus time histograms and firing rate histograms typically revealed only moderate orientation tuning in the ACtx (examples: Fig. $5 B, C$ ). For reference, we also recorded drifting grating responses from VCtx units. Side-by-side comparison of the most orientationselective ACtx and VCtx units shows a much higher degree of orientation selectivity in the VCtx (Fig. 5D,E). We calculated the OSI (see Materials and Methods) for all ACtx and VCtx SUs. We find strongly orientation-selective units in the VCtx (41\% [7 of 17] of OSIs $>0.75, n=7$ recordings in 3 mice), along with weakly tuned units, but only weakly tuned units in 
A

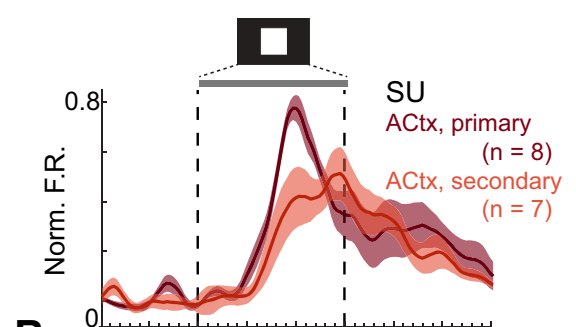

B

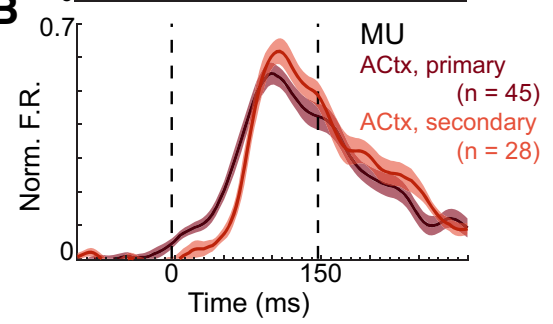

C

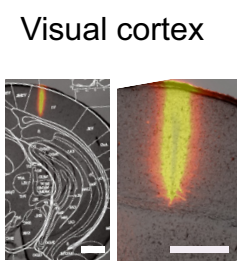

$\mathbf{E}$

ACtx vs. VCtx timing

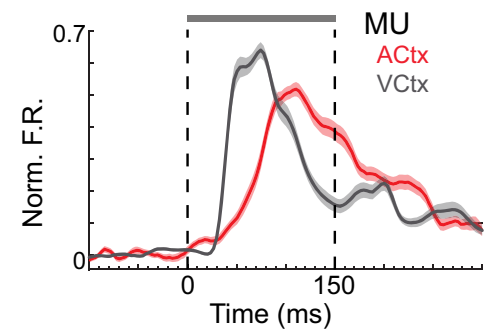

$\mathbf{F}$
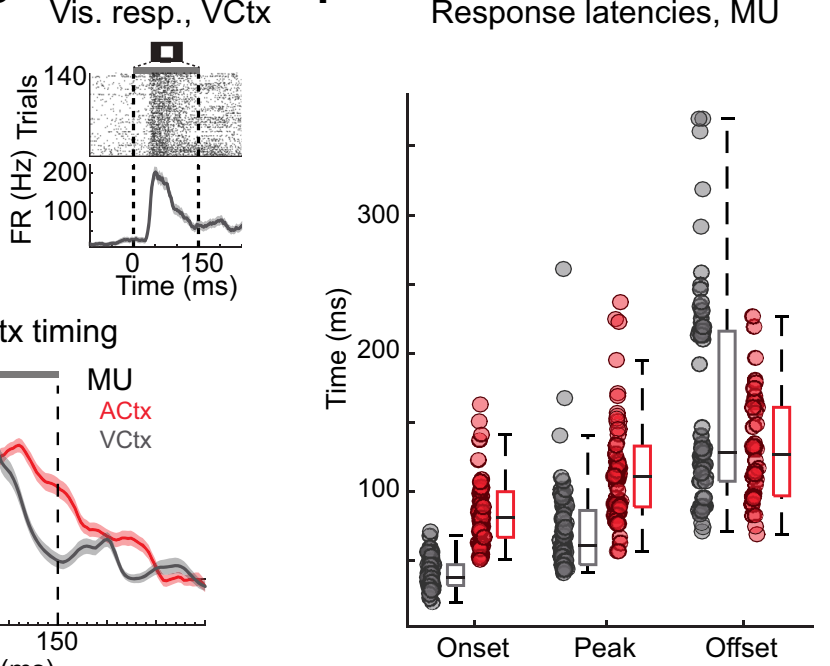

Figure 4. A, Mean normalized firing rate of visually responsive SUs ( $q=0.001$ following Benjamini-Hochberg false discovery rate correction) in primary (dark red represents $n=45 \mathrm{MUs}$ from 19 recordings in 12 mice) or secondary (light red represents $n=28$ MUs from 11 recordings in 8 mice) ACtx following a flash stimulus. $B$, Mean normalized firing rate of visually responsive MU sites in primary (dark red represents $n=45 \mathrm{MUs}$ from 19 recordings from 12 mice) or secondary (light red represents $n=28$ MUs from 11 recordings from 8 mice) ACtx following a flash stimulus. C, Example probe track of recording in VCtx. Scale bar, $500 \mu \mathrm{m}$. D. Example MU response to flash stimulus in VCtx. E, Comparison of response dynamics between ACtx and VCtx, including all significantly visually responsive MUs from both regions ( $n=73 \mathrm{ACtx}$ MU sites from 30 recordings in 15 mice; $n=78 \mathrm{VCtx}$ MU sites from 5 recordings from 3 mice). $F$, Latencies to onset, peak, and offset recorded from VCtx and ACtx, showing VCtx peak and onset responses occur earlier than those in ACtx, whereas offsets in VCtx occur later (see Results). In box plots, black line indicates median, box edges indicate 25 th and 75 th percentile, and whiskers extend to most extreme data points excluding outliers.

A

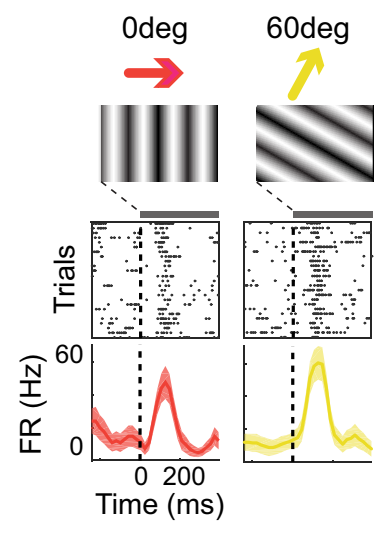

D ACtx, highest OSI

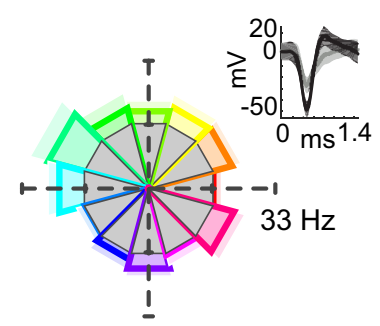

ACtx SU (example)
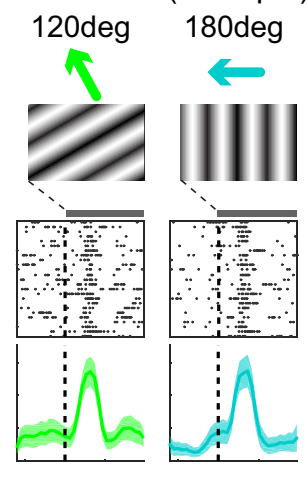

E

VCtx, highest OSI
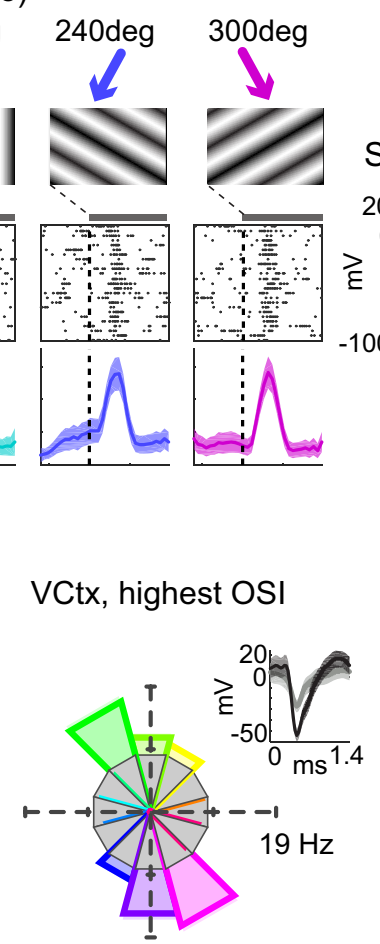

B

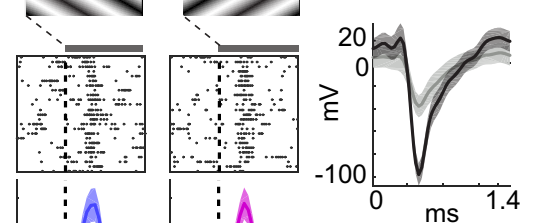

C

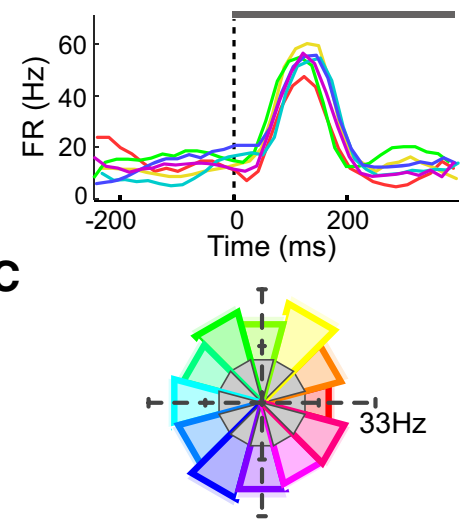

$\mathbf{F}$

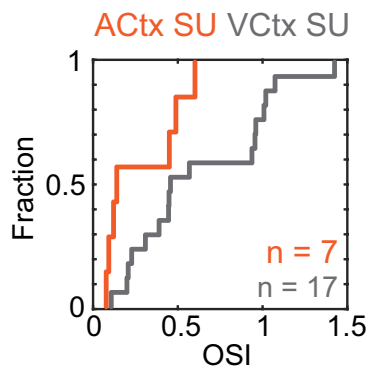

Figure 5. $A$, Example ACtx SU response to drifting gratings of varied orientations (colors). Right, SU waveform. $B$, All peristimulus time histogram responses from $A$ plotted together, revealing little preference for grating orientation. $\boldsymbol{C}$, Results in $\boldsymbol{B}$, visualized as baseline-normalized circular histogram. Colors represent visual response over 300 ms poststimulus window. Gray represents baseline firing rate. $\boldsymbol{D}$, ACtx unit with highest OSI. E, For comparison, VCtx unit with highest OSI. $\boldsymbol{F}$, Cumulative distribution plot of all OSIs, showing a higher degree of tuning in VCtx SUs $(n=17$ units from 7 recordings in 3 mice) than those in ACtx ( $n=7$ units from 5 recordings in 3 mice).

ACtx ( $0 \%$ [ 0 of 7 ] of OSIs $>0.75 ; n=5$ recordings in 4 mice; Fig. $5 F$ ); orientation selectivity differs significantly between these two populations (one-tailed Kolmogorov-Smirnov test statistic $=0.512 ; p=0.049)$. These results suggest that visual responses in ACtx do not carry fundamental visual information about edge orientation but instead may represent a more general signal indicating the presence and timing of a salient visual stimulus. 


\section{Discussion}

To determine whether mouse ACtx responds to visual stimulation, we presented awake mice with unimodal visual and auditory stimuli under passive conditions while performing acute recordings from auditory or visual cortices. In both primary and secondary ACtx, we found SU and MU activity that responded directly to visual flash and drifting grating stimuli in the absence of sound. These responses were almost entirely restricted to L6 and, to a lesser degree, L5. In L6 of ACtx, $\sim 25 \%$ of MUs were visually responsive; in L5, this value was $10 \%$; yet, $<2 \%$ of MUs in layers 2-4 were visually responsive. Visually responsive units in ACtx have longer latencies than those in VCtx and, unlike VCtx neurons, are not strongly tuned to drifting grating orientation. Together, these results suggest that the deep layers of cortex may represent a locus for cortical multisensory integration in the mouse.

These findings are supported by anatomical tracing work that shows that mouse primary ACtx receives inputs from VCtx (Banks et al., 2011). Anterograde tracers injected into secondary regions both lateral (V2L) and medial (V2M) of V1 reveal robust labeling of terminals in A1. Since this work, much has been done to further parcellate the fields of mouse secondary VCtx (e.g., Garrett et al., 2014); it remains to be tested whether projections to auditory areas vary further by visual cortical subfield. Particularly relevant here is the finding that neurons from V2 primarily send projections to L1 and L6 (Banks et al., 2011). This points to a potential anatomical pathway for visual signals to elicit spiking responses in the deep layers of mouse ACtx via monosynaptic connections from V2M and V2L. Although we did not observe visually responsive cells in L1, this layer of cortex was not well represented in this dataset ( $n=13 \mathrm{MU}$ sites recorded). Projections arriving at L1 do not necessarily target the sparse population of cells that reside there: the superficial visual projection to ACtx may terminate on the relatively large mass of L1 apical dendrites from pyramidal cells in L5 and L2/3 (Larkum and Zhu, 2002), and thus may produce some of the deep layer visual responses we observed. Further work involving methods such as transsynaptic tracing must be used to resolve questions of this nature.

Beyond direct connections from secondary visual cortices, there are several other potential pathways by which visual signals may drive spiking responses in the ACtx. Other areas of association cortex may send feedback projections to modulate processing in the ACtx. The gerbil primary ACtx receives direct input from multisensory cortical regions, such as posterior parietal cortex (Budinger et al., 2006). In addition, several thalamic regions with projections to ACtx show multimodal responses; the medial aspect of the medial geniculate body exhibits multisensory responses (Wepsic, 1966) and sends a dense projection to L6 of rat ACtx (Linke and Schwegler, 2000). Furthermore, the suprageniculate nucleus, another highly multimodal thalamic region, projects to L5 and L6 of rat ACtx (Smith et al., 2010). The termination patterns of these projections are also consistent with deep layer visual spiking responses; none of these anatomical pathways can be ruled out based on our results. While neurons in many visual stations, including primary and secondary visual cortices as well as visual thalamus, exhibit tuning to orientation, the untuned responses we observe in ACtx could result from the pooling of such tuned inputs.

The layer specificity of visually evoked spiking responses in ACtx may inform the role of such responses in modulating activity within the cortical column (Douglas et al., 1989). The deep or "infragranular" layers of cortex are considered the primary sub- cortical output layers but also send collaterals to cortical targets, including local circuitry. Historically, the role of L6, in particular, has been considered enigmatic due in part to its high degree of morphological and physiological heterogeneity (Briggs, 2010) and atypical sensory responses (Zhou et al., 2010). Recent work has shown that L6, through synapses onto local inhibitory interneurons, may play a role in gain control of sensory responses (Bortone et al., 2014). Furthermore, L6 neurons are known to influence cortical receptive field structure (Bolz and Gilbert, 1986) and gate sensory input through corticothalamic connections (Briggs and Usrey, 2008). Cells in these layers appear to be strategically located for sculpting and modulating sensory processing. The restriction of visually evoked spiking responses to the infragranular layers suggests that such modulation of sensory activity may be controlled, in part, by cross-modal inputs.

Previous work has shown that visually evoked spiking responses are rare in the ACtx. Kobayasi et al. (2013) recorded from A1 of the Mongolian gerbil, and concluded that 2 of their 128 units exhibited responses to a visual stimulus alone. In the ACtx of the ferret, reported percentages of visually responsive neurons are much higher, with $\sim 15 \%$ of primary auditory neurons showing responses to the light flash of an LED (Bizley et al., 2007). In the rat ACtx, $\sim 6 \%$ of units showed responses to the visual stimulus alone (Wallace et al., 2004). Although factors, such as crossspecies differences, likely explain some of these discrepancies in visual responsiveness, our work brings up the possibility that they may also be due, in part, to differences in laminar sampling. Our work also shows that visual responses are more prominent in secondary ACtx, consistent with findings in the ferret and monkey (Bizley et al., 2007; Kayser et al., 2008).

The findings presented here extend this literature by revealing that visual stimuli evoke spiking responses in the mouse ACtx and showing conclusively that visually responsive units show a strong laminar bias. Previous nonprimate work on audiovisual integration in the ACtx has largely been performed in anesthetized animals (Wallace et al., 2004; Bizley et al., 2007). Given that many anesthesias preferentially inhibit corticocortical connections (Raz et al., 2014) and that at least some visual information likely arrives at the ACtx through such connections (Banks et al., 2011), recordings in the awake animal may uncover responses otherwise obscured in anesthetized recordings. Furthermore, our examination of the orientation tuning properties of auditory responses begins to answer questions about the type of information visual responses convey to the local circuitry.

This work must be considered in the broader context of the literature on multisensory integration, much of which has found effects of cross-modal stimulation not in firing rate changes, but in evoked field potential responses and oscillatory changes (Ghazanfar et al., 2005; Lakatos et al., 2007). For example, Lakatos et al. (2007) found that visual stimulus onset resulted in phase reset of ongoing oscillations in the monkey ACtx with no change in MU spiking. It remains to be seen whether such a mechanism is also present in the mouse. Furthermore, there are additional aspects of the spiking signal in response to cross-modal stimulation that remain to be examined. The quenching of trial-to-trial response variability, for example, is a widespread phenomenon related to stimulus onset and may be an additional mechanism by which visual signals affect auditory cortical processing (Churchland et al., 2010).

This work is motivated partly by the utility of the mouse as a mammalian model organism for cell-type-specific microcircuit dissection. Tools such as fluorescent cell labeling, optogenetics, and chemogenetics, when applied to the problems of multisen- 
sory integration, may help elucidate the microcircuitry that integrates cross-modal signals. We hope that this study of visual responses in the ACtx of a genetic model organism will further the use of cell-type-specific tools for microcircuit dissection of multisensory phenomena.

\section{References}

Banks MI, Uhlrich DJ, Smith PH, Krause BM, Manning KA (2011) Descending projections from extrastriate visual cortex modulate responses of cells in primary auditory cortex. Cereb Cortex 21:2620-2638. CrossRef Medline

Benjamini Y, Hochberg Y (1995) Controlling the false discovery rate: a practical and powerful approach to multiple testing. J R Stat Soc B 57:289-300.

Bizley JK, Nodal FR, Bajo VM, Nelken I, King AJ (2007) Physiological and anatomical evidence for multisensory interactions in auditory cortex. Cereb Cortex 17:2172-2189. CrossRef Medline

Bolz J, Gilbert CD (1986) Generation of end-inhibition in the visual cortex via interlaminar connections. Nature 320:362-365. CrossRef Medline

Bortone DS, Olsen SR, Scanziani M (2014) Translaminar inhibitory cells recruited by layer 6 corticothalamic neurons suppress visual cortex. Neuron 82:474-485. CrossRef Medline

Briggs F (2010) Organizing principles of cortical layer 6. Front Neural Circuits 4:3. CrossRef Medline

Briggs F, Usrey WM (2008) Emerging views of corticothalamic function. Curr Opin Neurobiol 18:403-407. CrossRef Medline

Brosch M, Selezneva E, Scheich H (2005) Nonauditory events of a behavioral procedure activate auditory cortex of highly trained monkeys. J Neurosci 25:6797-6806. CrossRef Medline

Budinger E, Heil P, Hess A, Scheich H (2006) Multisensory processing via early cortical stages: connections of the primary auditory cortical field with other sensory systems. Neuroscience 143:1065-1083. CrossRef Medline

Buzsáki G (2004) Large-scale recording of neuronal ensembles. Nat Neurosci 7:446-451. CrossRef Medline

Calvert GA, Bullmore ET, Brammer MJ, Campbell R, Williams SC, McGuire PK, Woodruff PW, Iversen SD, David AS (1997) Activation of auditory cortex during silent lipreading. Science 276:593-596. CrossRef Medline

Carrasco A, Lomber SG (2011) Neuronal activation times to simple, complex, and natural sounds in cat primary and nonprimary auditory cortex. J Neurophysiol 106:1166-1178. CrossRef Medline

Churchland MM, Yu BM, Cunningham JP, Sugrue LP, Cohen MR, Corrado GS, Newsome WT, Clark AM, Hosseini P, Scott BB, Bradley DC, Smith MA, Kohn A, Movshon JA, Armstrong KM, Moore T, Chang SW, Snyder LH, Lisberger SG, Priebe NJ, et al. (2010) Stimulus onset quenches neural variability: a widespread cortical phenomenon. Nat Neurosci 13:369378. CrossRef Medline

Cook JD, Ellinwood EH Jr, Wilson WP (1968) Auditory habituation at primary cortex as a function of stimulus rate. Exp Neurol 21:167-175. CrossRef Medline

DiCarlo JJ, Lane JW, Hsiao SS, Johnson KO (1996) Marking microelectrode penetrations with fluorescent dyes. J Neurosci Methods 64:75-81. CrossRef Medline

Douglas RJ, Martin KAC, Whitteridge D (1989) A canonical microcircuit for neocortex. Neural Comput 1:480-488. CrossRef

Falchier A, Schroeder CE, Hackett TA, Lakatos P, Nascimento-Silva S, Ulbert I, Karmos G, Smiley JF (2010) Projection from visual areas V2 and prostriata to caudal auditory cortex in the monkey. Cereb Cortex 20:15291538. CrossRef Medline

Felleman DJ, Van Essen DC (1991) Distributed hierarchical processing in the primate cerebral cortex. Cereb Cortex 1:1-47. CrossRef Medline

Foxe JJ, Morocz IA, Murray MM, Higgins BA, Javitt DC, Schroeder CE (2000) Multisensory auditory-somatosensory interactions in early cortical processing revealed by high-density electrical mapping. Brain Res Cogn Brain Res 10:77-83. CrossRef Medline

Garrett ME, Nauhaus I, Marshel JH, Callaway EM (2014) Topography and areal organization of mouse visual cortex. J Neurosci 34:12587-12600. CrossRef Medline

Ghazanfar AA, Maier JX, Hoffman KL, Logothetis NK (2005) Multisensory integration of dynamic faces and voices in rhesus monkey auditory cortex. J Neurosci 25:5004-5012. CrossRef Medline

Gong S, Doughty M, Harbaugh CR, Cummins A, Hatten ME, Heintz N, Gerfen CR (2007) Targeting cre recombinase to specific neuron populations with bacterial artificial chromosome constructs. J Neurosci 27: 9817-9823. CrossRef Medline

Henschke JU, Noesselt T, Scheich H, Budinger E (2015) Possible anatomical pathways for short-latency multisensory integration processes in primary sensory cortices. Brain Struct Funct 220:955-977. CrossRef Medline

Iurilli G, Ghezzi D, Olcese U, Lassi G, Nazzaro C, Tonini R, Tucci V, Benfenati F, Medini P (2012) Sound-driven synaptic inhibition in primary visual cortex. Neuron 73:814-828. CrossRef Medline

Joachimsthaler B, Uhlmann M, Miller F, Ehret G, Kurt S (2014) Quantitative analysis of neuronal response properties in primary and higher-order auditory cortical fields of awake house mice (Mus musculus). Eur J Neurosci 39:904-918. CrossRef Medline

Kayser C, Petkov CI, Logothetis NK (2008) Visual modulation of neurons in auditory cortex. Cereb Cortex 18:1560-1574. CrossRef Medline

Kleiner M, Brainard D, Pelli D (2007) What's new in Psychtoolbox-3? ECVP Abstr Suppl.

Kobayasi KI, Suwa Y, Riquimaroux H (2013) Audiovisual integration in the primary auditory cortex of an awake rodent. Neurosci Lett 534:24-29. CrossRef Medline

Lakatos P, Chen CM, O'Connell MN, Mills A, Schroeder CE (2007) Neuronal oscillations and multisensory interaction in primary auditory cortex. Neuron 53:279-292. CrossRef Medline

Larkum ME, Zhu JJ (2002) Signaling of layer 1 and whisker-evoked $\mathrm{Ca}^{2+}$ and $\mathrm{Na}^{+}$action potentials in distal and terminal dendrites of rat neocortical pyramidal neurons in vitro and in vivo. J Neurosci 22:6991-7005. Medline

Lee E, Hong J, Park YG, Chae S, Kim Y, Kim D (2015) Left brain cortical activity modulates stress effects on social behavior. Sci Rep 5:13342. CrossRef Medline

Linke R, Schwegler H (2000) Convergent and complementary projections of the caudal paralaminar thalamic nuclei to rat temporal and insular cortex. Cereb Cortex 10:753-771. CrossRef Medline

McGurk H, MacDonald J (1976) Hearing lips and seeing voices. Nature 264:746-748. CrossRef Medline

Niell CM, Stryker MP (2008) Highly selective receptive fields in mouse visual cortex. J Neurosci 28:7520-7536. CrossRef Medline

Olsen SR, Bortone DS, Adesnik H, Scanziani M (2012) Gain control by layer six in cortical circuits of vision. Nature 483:47-52. CrossRef Medline

Paxinos G, Franklin KB (2004) The mouse brain in stereotaxic coordinates. San Diego, CA: Academic.

Raz A, Grady SM, Krause BM, Uhlrich DJ, Manning KA, Banks MI (2014) Preferential effect of isoflurane on top-down vs. bottom-up pathways in sensory cortex. Front Syst Neurosci 8:191. CrossRef Medline

Schaefer M, Flor H, Heinze HJ, Rotte M (2006) Dynamic modulation of the primary somatosensory cortex during seeing and feeling a touched hand. Neuroimage 29:587-592. CrossRef Medline

Skipper JI, van Wassenhove V, Nusbaum HC, Small SL (2007) Hearing lips and seeing voices: how cortical areas supporting speech production mediate audiovisual speech perception. Cereb Cortex 17:2387-2399. CrossRef Medline

Smith PH, Manning KA, Uhlrich DJ (2010) Evaluation of inputs to rat primary auditory cortex from the suprageniculate nucleus and extrastriate visual cortex. J Comp Neurol 518:3679-3700. CrossRef Medline

Stiebler I, Neulist R, Fichtel I, Ehret G (1997) The auditory cortex of the house mouse: left-right differences, tonotopic organization and quantitative analysis of frequency representation. J Comp Physiol A 181:559-571. CrossRef Medline

Wallace MT, Ramachandran R, Stein BE (2004) A revised view of sensory cortical parcellation. Proc Natl Acad Sci U S A 101:2167-2172. CrossRef Medline

Wepsic JG (1966) Multimodal sensory activation of cells in the magnocellular medial geniculate nucleus. Exp Neurol 15:299-318. CrossRef Medline

Zhou Y, Liu BH, Wu GK, Kim YJ, Xiao Z, Tao HW, Zhang LI (2010) Preceding inhibition silences layer 6 neurons in auditory cortex. Neuron 65:706-717. CrossRef Medline 